\title{
Colour-blind can distinguish colour pallets
}

\author{
Jakub Przybyło* \\ AGH University of Science and Technology \\ al. A. Mickiewicza 30 \\ 30-059 Krakow, Poland \\ przybylo@wms.mat.agh.edu.pl
}

Submitted: Sep 24, 2013; Accepted: Apr 3, 2014; Published: May 2, 2014

Mathematics Subject Classifications: 05C15

\begin{abstract}
Let $c: E \rightarrow\{1, \ldots, k\}$ be an edge colouring of a connected graph $G=(V, E)$. Each vertex $v$ is endowed with a naturally defined pallet under $c$, understood as the multiset of colours incident with $v$. If $\delta(G) \geqslant 2$, we obviously (for $k$ large enough) may colour the edges of $G$ so that adjacent vertices are distinguished by their pallets of colours. Suppose then that our coloured graph is examined by a person who is unable to name colours, but perceives if two object placed next to each other are coloured differently. Can we colour $G$ so that this individual can distinguish colour pallets of adjacent vertices? It is proved that if $\delta(G)$ is large enough, then it is possible using just colours 1,2 and 3. This result is sharp and improves all earlier ones. It also constitutes a strengthening of a result by Addario-Berry, Aldred, Dalal and Reed (2005).
\end{abstract}

Keywords: Neighbour distinguishing colouring; Colour pallet; Colour-blind.

\section{Results}

Consider a simple graph $G=(V, E)$. Given vertex $v \in V$, we denote by $d(v)$ the degree of $v$, and by $N(v)$ the neighbourhood of $v$ in $G$. To be precise, we shall also use the notation $d_{G}(v)$ and $N_{G}(v)$, resp., at times. A not necessarily proper edge colouring $c$ : $E \rightarrow\{1,2, \ldots, k\}$ is called neighbour distinguishing (or vertex colouring, see e.g. [1]) if for every edge $u v \in E$, the multiset of colours incident with $u$ is distinct from the multiset of colours incident with $v$. The problem of finding minimum $k$ so that every graph without a $K_{2}$ component admits a neighbour distinguishing edge colouring with $k$ colours first arose

${ }^{*}$ Research partly supported by the Polish Ministry of Science and Higher Education and supported by the National Science Centre grant no. DEC-2011/01/D/ST1/04154. 
in the paper of Karoński, Łuczak and Thomason [6] as a tool developed for making some progress concerning now well known 1-2-3 Conjecture, cf. [5] for the most recent result regarding this. They proved that $k \leqslant 183$ always suffice, or even $k \leqslant 30$ if the minimum degree $\delta$ of $G$ is at least $10^{99}$. This was then greatly improved by Addario-Berry et al. [1], who showed that four colours are sufficient and provided the following refinement for graphs of sufficiently large minimum degree.

Theorem 1. ([1]) Every graph of minimum degree $\delta \geqslant 1000$ and without a $K_{2}$ component admits a neighbour distinguishing colouring with 1, 2 and 3.

For every vertex $v$ and a colouring $c: E \rightarrow\{1,2, \ldots, k\}$, set $\bar{c}(v)=\left(a_{1}, \ldots, a_{k}\right)$, where $a_{i}=\left|c^{-1}(\{i\})\right|$ for $i=1, \ldots, k$. Thus $c$ is neighbour distinguishing if $\bar{c}(u)$ and $\bar{c}(v)$ differ in at least one coordinate for every edge $u v \in E$. Let us strengthen this requirement as follows: For every $v$, let us re-order $\bar{c}(v)$ non-decreasingly and denote the outcome by $c^{*}(v)=\left(d_{1}, \ldots, d_{k}\right)$, hence $0 \leqslant d_{1} \leqslant d_{2} \leqslant \ldots \leqslant d_{k}$ and $\sum_{i=1}^{k} d_{i}=d(v)$. We say that a colour-blind person can distinguish neighbours under colouring $c$ if for every edge $u v \in E, c^{*}(u) \neq c^{*}(v)$ (hence also $\bar{c}(u) \neq \bar{c}(v)$ ). Here we consider a peculiar kind of colour-blindness a person suffering from which cannot name colours, but is able to state whether two objects (e.g. edges) placed next to each other are coloured the same or differently. Therefore they are able to group and count subsets of monocoloured edges incident with $v$, but cannot associate these quantities to the specific colours, and thus $c^{*}(v)$ represents the most comprehensive information they can deliver on the frequencies of colours surrounding $v$. The smallest integer $k$ for which such colouring exists is called the colour-blind index of $G$, and is denoted by $\operatorname{dal}(G)$. This notion refers to the English chemist John Dalton, who in 1798 wrote the first paper on colour-blindness. In fact, because of Dalton's work, the condition is often called daltonism.

There are graphs for which this parameter is not defined. All known examples of such graphs have minimum degree at most 3 . It is thus believed that the following conjecture should hold with $\delta_{0}=4$.

Conjecture 2. ([4]) There exists $\delta_{0}$ such that dal $(G)$ is defined for every graph $G$ with $\delta(G) \geqslant \delta_{0}$.

Moreover, it has been conjectured and proven the following.

Conjecture 3. ([4]) There exists a constant $K$ such that dal $(G) \leqslant K$ for every graph $G$ for which $\operatorname{dal}(G)$ exists.

Theorem 4. ([4]) For every $R>1$, there exists $\delta_{0}$ such that if $G$ is any graph with minimum degree $\delta(G) \geqslant \delta_{0}$ and maximum degree $\Delta(G) \leqslant R \delta(G)$, then

$$
\operatorname{dal}(G) \leqslant 6
$$

Theorem 5. ([4]) For every d-regular graph $G$ of degree $d \geqslant 2 \cdot 10^{7}$,

$$
\operatorname{dal}(G) \leqslant 6
$$


Following the probabilistic approach from [4], Przybyło proved then that in the case of regular graphs one may significantly reduce the threshold for $d$ at the cost of a few more colours.

Theorem 6. ([7]) For every d-regular graph $G$ of degree $d \geqslant 960$,

$$
\operatorname{dal}(G) \leqslant 15 \text {. }
$$

In this note we shall prove the following improvement of Theorems 4, 5 and 6 , which is a strengthening of Theorem 1 as well. Note that this also proves Conjecture 2 to hold.

Theorem 7. For each graph $G$ with $\delta(G) \geqslant 3462$,

$$
\operatorname{dal}(G) \leqslant 3
$$

Note that this upper bound is tight, since e.g. dal $\left(K_{n}\right)=3$ for every $n \geqslant 7$, see [4] for details.

\section{Proofs}

We shall use the following theorem from [3], which develops similar ideas included in $[1,2]$.

Theorem 8. ([3]) Given a graph $G=(V, E)$ and for every $v \in V$, integers $a_{v}^{-}, a_{v}^{+}$such that $a_{v}^{-} \leqslant\left\lfloor\frac{d(v)}{2}\right\rfloor \leqslant a_{v}^{+}<d(v)$ and

$$
a_{v}^{+} \leqslant \min \left\{\frac{d(v)+a_{v}^{-}}{2}+1,2 a_{v}^{-}+3\right\},
$$

there exists a spanning subgraph $H$ of $G$ such that $d_{H}(v) \in\left\{a_{v}^{-}, a_{v}^{-}+1, a_{v}^{+}, a_{v}^{+}+1\right\}$ for each $v \in V$.

Corollary 9. For each integer $d, d>60$, there exist a list $I_{d}$ of at least $\frac{d-57}{4}$ consecutive integers and a divisible by 7 integer $h_{d}, h_{d}>\left|I_{d}\right|$, such that for any graph $G=(V, E)$ with minimum degree $\delta>60$ and any list of integers $\left(a_{v}\right)_{v \in V}$ with $a_{v} \in I_{d(v)}$ for each $v \in V$, there exists a spanning subgraph $H$ of $G$ such that $d_{H}(v) \in\left\{a_{v}, a_{v}+1, a_{v}+h_{d(v)}, a_{v}+\right.$ $\left.h_{d(v)}+1\right\}$ for every $v \in V$.

Proof. We shall prove that the lists $I_{d}:=\left\{\left\lceil\frac{d}{4}\right\rceil+3, \ldots,\left\lfloor\frac{d}{2}\right\rfloor-11\right\}$, where

$$
\left|I_{d}\right|=\left(\left\lfloor\frac{d}{2}\right\rfloor-11\right)-\left(\left\lceil\frac{d}{4}\right\rceil+3\right)+1 \geqslant \frac{d-57}{4},
$$

and $h_{d}$ defined as the least integer divisible by 7 not smaller than $\left\lceil\frac{d}{4}\right\rceil$ fulfill our requirements. It is then sufficient to prove that for a given vertex $v$ with $d(v)>60$, any pair of integers $a_{v}^{-} \in I_{d(v)}$ and $a_{v}^{+}:=a_{v}^{-}+h_{d(v)}$ comply with the requirements of Theorem 8 . 
For any such $a_{v}^{-}$and $h_{d(v)}$ note first that by definition,

$$
\left\lceil\frac{d(v)}{4}\right\rceil \leqslant h_{d(v)} \leqslant\left\lceil\frac{d(v)}{4}\right\rceil+6 .
$$

Clearly $a_{v}^{-} \leqslant\left\lfloor\frac{d(v)}{2}\right\rfloor$ and

$$
\left\lfloor\frac{d(v)}{2}\right\rfloor \leqslant a_{v}^{+}=a_{v}^{-}+h_{d(v)}<d(v)
$$

hence it is enough to prove inequality (1) for our $a_{v}^{-}$and $a_{v}^{+}$. Note then that

$$
a_{v}^{+}=a_{v}^{-}+h_{d(v)} \leqslant a_{v}^{-}+\left\lceil\frac{d(v)}{4}\right\rceil+6=a_{v}^{-}+\left(\left\lceil\frac{d(v)}{4}\right\rceil+3\right)+3 \leqslant 2 a_{v}^{-}+3
$$

and analogously,

$$
\begin{aligned}
a_{v}^{+} & \leqslant \frac{a_{v}^{-}}{2}+\frac{a_{v}^{-}}{2}+\left\lceil\frac{d(v)}{4}\right\rceil+5+1 \\
& \leqslant \frac{a_{v}^{-}}{2}+\left(\frac{\left\lfloor\frac{d(v)}{2}\right\rfloor-11}{2}+\left\lceil\frac{d(v)}{4}\right\rceil+5\right)+1 \leqslant \frac{a_{v}^{-}}{2}+\frac{d(v)}{2}+1,
\end{aligned}
$$

thus (1) holds.

Note that the elements of each set $\left\{a_{v}, a_{v}+1, a_{v}+h_{d(v)}, a_{v}+h_{d(v)}+1\right\}$ from the corollary above have only two (consecutive) remainders modulo 7 .

Observation 10. For any $c \in \mathbb{Z}_{7}$ there exist $a, b \in \mathbb{Z}_{7}$ such that the sets $A:=\{a, a+1\}$, $B:=\{b, b+1\}$ and $C:=\{c-x-y: x \in A, y \in B\}$ (additions modulo 7) form a partition of $\mathbb{Z}_{7}$.

Proof. Note that $C=\{c-a-b-2, c-a-b-1, c-a-b\}$. For every $c=3 p+1, p \in \mathbb{Z}_{7}$, it is thus sufficient to take $a=p$ and $b=p+2$, since then $A=\{p, p+1\}, B=\{p+2, p+3\}$ and $C=\{p+4, p+5, p+6\}$ in $\mathbb{Z}_{7}$.

Note also that the number 7 cannot be replaced by a smaller integer in the observation above (if we require $A, B$ and $C$ to be disjoint).

Proof of Theorem 7. We shall colour our graph using the colours 1, 2 and 3. First we shall choose edges to be coloured with 1 . These will form a subgraph $H_{1}$ of $G$. Then we shall choose some subgraph $H_{2}$ of the graph $G^{\prime}=\left(V, E \backslash E\left(H_{1}\right)\right)$ obtained by removing the edges of $H_{1}$ from $G$, and colour the edges of $H_{2}$ with 2. The remaining edges will receive colour 3 .

Note that any two neighbours may not be distinguishable in the obtained colouring only if they share the same degree. For a given degree $d \geqslant 3462$ with remainder $c$ modulo 
7 , let $\mathbb{Z}_{7}=A_{d} \cup B_{d} \cup C_{d}$ be a partition guaranteed by Observation 10 , and let $I_{d}$ be a set of integers from Corollary $9,\left|I_{d}\right| \geqslant \frac{d-57}{4}$. We shall ensure that the subgraphs $H_{1}$ and $H_{2}$ are chosen so that $d_{H_{1}}(v)(\bmod 7)$ belongs to $A_{d(v)}, d_{H_{2}}(v)(\bmod 7)$ belongs to $B_{d(v)}$, and hence $d(v)-d_{H_{1}}(v)-d_{H_{2}}(v)(\bmod 7)$ belongs to $C_{d(v)}$. Then two neighbours will not be distinguishable for a colour-blind person only if they have exactly the same number of incident edges in each of the colours.

Let us fix any ordering of the vertices of $G$ into a sequence $v_{1}, v_{2}, \ldots, v_{n}$. One after another we shall choose $a_{v_{i}}$ for every $v_{i}$ in order to apply Corollary 9. Suppose that $v_{i}$ $(1 \leqslant i \leqslant n)$ has degree $d, A_{d}=\{a, a+1\}$, and we have already chosen $a_{v_{j}}$ for each vertex $v_{j}$ with $j<i$. We then choose $a_{v_{i}} \in I_{d}$ greedily so that $a_{v_{i}} \equiv a(\bmod 7)$ and the number of backward neighbours $v_{j}$ of $v_{i}$, i.e. $v_{j} \in N\left(v_{i}\right)$ with $j<i$, of degree $d$ and with $a_{v_{j}}=a_{v_{i}}$ is as small as possible. Denote this minimal subset of backward neighbours of $v_{i}$ by $B\left(v_{i}\right)$. In particular we may have $\left|B\left(v_{i}\right)\right|=0$. Note that we have at least $\left\lfloor\frac{I_{d}}{7}\right\rfloor$ choices for $a_{v_{i}}$ in $I_{d}$ such that $a_{v_{i}} \equiv a(\bmod 7)$. Moreover

$$
29\left\lfloor\frac{I_{d}}{7}\right\rfloor \geqslant 29\left\lfloor\frac{d-57}{28}\right\rfloor \geqslant 29\left(\frac{d-57}{28}-\frac{27}{28}\right)=d+1+\frac{d-2464}{28} \geqslant d+1,
$$

and thus by the pigeonhole principle, $a_{v_{i}}$ can be chosen so that $\left|B\left(v_{i}\right) \cup\left\{v_{i}\right\}\right| \leqslant 29$, hence $\left|B\left(v_{i}\right)\right| \leqslant 28$. For $a_{v_{1}}, \ldots, a_{v_{n}}$ chosen in this manner, Corollary 9 guarantees the existence of a subgraph $H_{1}$ of $G$ such that $d_{H_{1}}(v) \in\left\{a_{v}, a_{v}+1, a_{v}+h_{d(v)}, a_{v}+h_{d(v)}+1\right\}$ for each $v \in V$, where $h_{d(v)}$ 's are the divisible by 7 constants from Corollary 9. Moreover, since for each $d$ we have $h_{d}>\left|I_{d}\right|$, then for a given vertex $v$ and its backward neighbour $w$, both of degree $d$, we may have $d_{H_{1}}(v)=d_{H_{1}}(w)$ only if $w \in B(v)$.

Let us now consider the graph $G^{\prime}$ that was left of $G$ after removing the edges of $H_{1}$. We may assume that we have used $I_{d}$ and $h_{d}$ defined in the first three lines of the proof of Corollary 9, hence for each $v \in V$ of degree $d$ in $G$,

$$
d_{H_{1}}(v) \leqslant a_{v}+h_{d(v)}+1 \leqslant\left\lfloor\frac{d}{2}\right\rfloor-11+\left\lceil\frac{d}{4}\right\rceil+6+1=\left\lfloor\frac{d}{2}\right\rfloor+\left\lceil\frac{d}{4}\right\rceil-4 \leqslant \frac{3}{4} d-\frac{7}{2} .
$$

Consequently,

$$
\delta\left(G^{\prime}\right) \geqslant\left\lceil\frac{1}{4} \delta(G)+\frac{7}{2}\right\rceil \geqslant 869 .
$$

In order to again apply Corollary 9 , this time to $G^{\prime}$, one after another we choose $b_{v_{i}}$ for each vertex $v_{i}$ in the fixed ordering. Suppose that $v_{i}(1 \leqslant i \leqslant n)$ had degree $d$ in $G$, $B_{d}=\{b, b+1\}$, and we have already chosen $b_{v_{j}}$ for each vertex $v_{j}$ with $j<i$. Denote by $B^{\prime}\left(v_{i}\right)$ the subset of those vertices $w$ from $B\left(v_{i}\right)$ for which $d_{H_{1}}(w)=d_{H_{1}}(v)$, hence $\left|B^{\prime}\left(v_{i}\right)\right| \leqslant\left|B\left(v_{i}\right)\right| \leqslant 28$. We then choose $b_{v_{i}} \in I_{d_{G^{\prime}}}\left(v_{i}\right)$ greedily so that $b_{v_{i}} \equiv b(\bmod 7)$ and $b_{v_{i}} \neq b_{v_{j}}$ for each $v_{j} \in B^{\prime}\left(v_{i}\right)$. Since $\left|B^{\prime}\left(v_{i}\right)\right| \leqslant 28$ and

$$
\left|I_{d_{G^{\prime}}\left(v_{i}\right)}\right| \geqslant \frac{d_{G^{\prime}}\left(v_{i}\right)-57}{4} \geqslant \frac{869-57}{4}=29 \cdot 7
$$

such choice is always possible. Then, by Corollary 9, there exists a subgraph $\mathrm{H}_{2}$ of $G^{\prime}$ with $d_{H_{2}}(v) \in\left\{b_{v}, b_{v}+1, b_{v}+h_{d_{G^{\prime}}(v)}, b_{v}+h_{d_{G^{\prime}}(v)}+1\right\}$ for each vertex $v \in V$. Note then that for every $v \in V$ and $w \in B^{\prime}(v), d_{G^{\prime}}(v)=d_{G^{\prime}}(w)$, and thus $d_{H_{2}}(v) \neq d_{H_{2}}(w)$. 
As mentioned, we colour each edge of $H_{1}$ with 1 , each edge of $H_{2}$ with 2 , and the remaining edges receive colour 3. Additionally, by our construction, for every vertex $v \in V$ we have $d_{H_{1}}(v)(\bmod 7) \in A_{d(v)}, d_{H_{2}}(v)(\bmod 7) \in B_{d(v)}$ and $d(v)-d_{H_{1}}(v)-d_{H_{2}}(v)$ $(\bmod 7) \in C_{d(v)}$, where $A_{d(v)} \cup B_{d(v)} \cup C_{d(v)}$ constitutes a partition of $\mathbb{Z}_{7}$. For each edge $v_{j} v_{i} \in E$ with $j<i$ and $d\left(v_{i}\right)=d\left(v_{j}\right)=d$, if $v_{j} \notin B^{\prime}\left(v_{i}\right)$, then $v_{i}$ and $v_{j}$ are distinguishable by colour 1 , and in the remaining cases by colour 2 .

\section{Concluding Remarks}

Intriguingly, there is still lack of complete characterization of the family of graphs with well defined 'dal' parameter. Thus in general it is not known if a finite bound for the graph invariant in question exists for these graphs, cf. Conjecture 3. Surprisingly vertices with 'smaller' degree appear more difficult to handle, and seem to require a different approach.

\section{References}

[1] L. Addario-Berry, R. E. L. Aldred, K. Dalal, B. A. Reed. Vertex colouring edge partitions. J. Combin. Theory Ser. B, 94(2):237-244, 2005.

[2] L. Addario-Berry, K. Dalal, C. McDiarmid, B. A. Reed, A. Thomason. VertexColouring Edge-Weightings. Combinatorica, 27(1):1-12, 2007.

[3] L. Addario-Berry, K. Dalal, B. A. Reed. Degree Constrained Subgraphs. Discrete Appl. Math., 156(7):1168-1174, 2008.

[4] R. Kalinowski, M. Pilśniak, J. Przybyło, M. Woźniak. Can colour-blind distinguish colour pallets? Electron. J. Combin., 20(3):\#P23, 2013.

[5] M. Kalkowski M. Karoński, F. Pfender. Vertex-coloring edge-weightings: Towards the 1-2-3 conjecture. J. Combin. Theory Ser. B, 100:347-349, 2010.

[6] M. Karoński, T. Łuczak, A. Thomason. Edge weights and vertex colours. J. Combin. Theory Ser. B, 91:151-157, 2004.

[7] J. Przybyło. On colour-blind distinguishing colour pallets in regular graphs. J. Comb. Optim., to appear. doi:10.1007/s10878-012-9556-x 